\title{
Attribute Reduction for Generalized Decision Systems*
}

\author{
Bi-Jun REN, Yan-Ling FU \\ Department of Information Engineering \\ Henan College of Finance and Taxation \\ Zhengzhou, Henan 451464, China
}

\author{
Ke-Yun QIN \\ College of Mathematics \\ Southwest Jiaotong University \\ Chengdu, Sichuan 610031, China
}

\begin{abstract}
Attribute reduction of information system is one of the most important applications of rough set theory. This paper focuses on generalized decision system and aims at studying positive region reduction and distribution reduction based on generalized indiscernibility relation. The judgment theorems for attribute reductions and attribute reduction approaches are presented. Our approaches improved the existed discernibility matrix and discernibility conditions. Furthermore, the reduction algorithms based on discernible degree are proposed.
\end{abstract}

Keywords-Rough set; generalized indiscernibility relation; positive region reduction; distribution reduction

\section{INTRODUCTION}

The theory of rough sets, proposed by Pawlak[6], is an extension of the set theory. Rough set theory has been conceived as a tool to conceptualize, organize, and analyze various types of data, in particular, to deal with inexact, uncertain or vague knowledge in applications related to artificial intelligence.

Information systems (sometimes called data tables, attribute-value systems, decision system etc.) are used for representing knowledge. A basic problem related to many practical applications of information systems is whether the whole set of attributes is always necessary to define a given partition of a universe. This problem is referred to as knowledge reduction, i.e., removing superfluous attributes from the information systems in such a way that the remaining attributes are the most informative. A large variety of approaches have been proposed in the literatures for effective and efficient reduction of knowledge. Of all paradigms, rough set theory is perhaps the most recent one making significant contribution to the field. Based on this theory and discernibility functions, some approaches for attribute reduction in complete and discrete decision systems are proposed[5,9,11,14,16].

In many practical situations, it may happen that the precise values of some of the attributes in an information system are not known, i.e. are missing or known partially. Such a system is called an incomplete information system. In order to deal with incomplete information systems, classical rough sets have been extended to several general models by using other binary relations or covers on the universe $[1,2,7,8,10,15,18,19]$. Based on these extended rough set models, the researchers have put forward several

This work has been supported by the National Natural Science Foundation of China (Grant No. 61473239), The Key Scientific and Technological Funds (Grant No. 142102310096) of Henan, China and Soft-scientific Item (Grant No. 142400410671) of Henan, China. meaningful indiscernibility relations in incomplete information system to characterize the similarity of objects. For instance, Kryszkiewicz[3,4] introduced a kind of indiscernibility relation, called tolerance relation, to handle incomplete information tables. Stefanowski[12] introduced two generalizations of the rough sets theory to handle the missing value. The first generalization introduces the use of a non symmetric similarity relation in order to formalize the idea of absent value semantics. The second proposal is based on the use of valued tolerance relations. The tolerance relation has also been generalized to constrained similarity relation and constrained dissymmetrical similarity relation[2,13,17]. Accordingly, some attribute reduction approached for incomplete decision systems have been proposed. In this paper, an approach to attribute reduction for incomplete decision systems based on generalized indiscernibility relation is presented. Specifically, this study is not limited to a particular indiscernibility relation, but focus on the indiscernibility relation that satisfies reflexivity and symmetry. A general theory frame of attribute reduction for incomplete decision system will be presented. The paper is organized as follows: In Section 2, we recall some notions and properties of rough sets and decision systems. In Section 3, we propose an approach for positive region reduction. The reduction algorithm based on discernible degree is also presented. Section 4 is devoted to distribution reduction. The paper is completed with some concluding remarks.

\section{GENERALIZED DECISION SYSTEMS}

An information system is a triplet $(U, A, F)$, where $U$ is a nonempty finite set of objects called the universe of discourse, $A=\left\{a_{1}, \cdots, a_{m}\right\}$ is a nonempty finite set of attributes, $F=\left\{f_{j} ; j \leq m\right\}$ is a set of information functions such that $f_{j}(x) \in V_{j}$ for all $x \in U$, where $V_{j}$ is the domain of attribute $a_{j}$ . A decision system $(U, C \cup\{d\}, F)$ is a special case of an information system, where $d$ is a special attribute called decision. The elements of $C$ are called conditional attributes.

In a generalized decision system, we do not care about the information function, but focus on the indiscernibility relations generated by attributes. Concretely, a generalized decision system is a triple $S=(U, A, d)$, where $U$ is a nonempty universe of objects, $A$ is a set of conditional attributes, and $d$ is a distinguished decision attribute. Each conditional attribute $a$ determines an indiscernibility relation which is denoted by $R_{a}$. 
In what follows we suppose that $R_{a}$ is reflexive. Additionally, the decision attribute $d$ determine a partition $U / d=\left\{D_{1}, \cdots, D_{r}\right\}$ of $U$. If $x \in D_{i}$, then we take $i$ as the decision value of $x$ and denoted by $d(x)=i$.

Let $S=(U, A, d)$ be a generalized decision system. For any $B \subseteq A$, the indiscernibility relation generated by $B$ is defined as $R_{B}=\cap_{a \in B} R_{a}$. For $x \in U$, the neighborhood of $x$ related to $R_{B}$ is denoted as $R_{B}(x)=\left\{y \in U ;(x, y) \in R_{B}\right\} \quad$. Obviously, $R_{B}(x)=\cap_{a \in B} R_{a}(x)$. Additionally, because of the reflexivity of $R_{B},\left\{R_{B}(x) ; x \in U\right\}$ forms a cover of $U$.

Definition 2.1[2,15] Let $S=(U, A, d)$ be a generalized decision system. For any $B \subseteq A, X \subseteq U$, the lower approximation and upper approximation of $X$ with respect to $R_{B}$ are defined as

$$
\begin{aligned}
& \underline{R_{B}}(X)=\left\{x \in U ; R_{B}(x) \subseteq X\right\} \\
& \overline{R_{B}}(X)=\left\{x \in U ; R_{B}(x) \cap X \neq \varnothing\right\}
\end{aligned}
$$

Theorem 2.1[2,15] Let $S=(U, A, d)$ be a generalized decision system, $B \subseteq A, X, Y \subseteq U$. Then

(1) $\underline{R_{B}}(X) \subseteq X \subseteq \overline{R_{B}}(X)$.

(2)If $X \subseteq Y$, then $\underline{R_{B}}(X) \subseteq \underline{R_{B}}(Y), \overline{R_{B}}(X) \subseteq \overline{R_{B}}(Y)$.

(3) $\underline{R_{B}}(X \cap Y)=\underline{R_{B}}(X) \cap \underline{R_{B}}(Y), \overline{R_{B}}(X \cup Y) \subseteq \overline{R_{B}}(X) \cup \overline{R_{B}}(Y)$.

(4) $\overline{R_{B}}(X)=\sim \underline{R_{B}}(\sim X), \underline{R_{B}}(X)=\sim \overline{R_{B}}(\sim X)$.

\section{AtTRIBUte REDUCTION BASED ON Positive REGION}

The section is devoted to the discussion of positive region reduction of generalized decision systems.

Definition 3.1[11] Let $S=(U, A, d)$ be a generalized decision system, $B \subseteq A, U / d=\left\{D_{1}, \cdots, D_{r}\right\}$. The positive region of $d$ with respect to $B$ is defined as

$$
\operatorname{Pos}_{B}(d)=\underset{X \in U / d}{\cup} \underline{R_{B}}(X)=\cup \operatorname{is}_{B} \underline{R_{B}}\left(D_{i}\right)
$$

The above definition shows that $x \in \operatorname{Pos}_{B}(d)$ if and only if the objects in $R_{B}(x)$ have the same decision values. Thus, $\operatorname{Pos}_{B}(d)$ is the set of all elements of $U$ that can be uniquely classified to blocks of the partition $U / d$ by means of $B$. If we take $B$ as the set of conditional attributes, then $x \in \operatorname{Pos}_{B}(d)$ means the decision rule with respect to $x$ is definite.

Theorem 3.1[9] Let $S=(U, A, d)$ be a generalized decision system, $B \subseteq A, U / d=\left\{D_{1}, \cdots, D_{r}\right\}$. Then

(1) $\operatorname{Pos}_{B}(d) \subseteq \operatorname{Pos}_{A}(d)$.

(2) $\operatorname{Pos}_{B}(d)=\operatorname{Pos}_{A}(d)$ if and only if $\underline{R_{B}}\left(D_{i}\right)=\underline{R_{A}}\left(D_{i}\right)$ for each $i \leq r$.
(3) $x \in \operatorname{Pos}_{B}(d)$ if and only if $x \in \underline{R_{B}}\left([x]_{d}\right)$.

Definition 3.2 Let $S=(U, A, d)$ be a generalized decision system. If $B \subseteq A$ such that $\operatorname{Pos}_{B}(d)=\operatorname{Pos}_{A}(d)$, then $B$ is called a positive region consistent set of $S$. The minimal positive region consistent set of $S$ (with respect to set inclusion relation) is called as positive region reduction of $S$.

Let $S=(U, A, d)$ be a generalized decision system, $x, y \in U$. We consider the following condition $\omega(x, y)$ :

$$
\omega(x, y): x \in \operatorname{Pos}_{A}(d) \wedge d(x) \neq d(y) .
$$

We note that $\omega(x, y)$ is not symmetric to $x$ and $y$.

Theorem 3.2[9] Let $S=(U, A, d)$ be a generalized decision system. If $x, y \in U$ satisfy $\omega(x, y)$, then $\alpha_{A}(x, y) \neq \varnothing$, where $\alpha_{A}(x, y)=\left\{a \in A ;(x, y) \notin R_{a}\right\}$.

Theorem 3.3 Let $S=(U, A, d)$ be a generalized decision system, $B \subseteq A . B$ is a positive region consistent set of $S$ if and only if $B \cap \alpha_{A}(x, y) \neq \varnothing$ for $x, y \in U$ satisfy $\omega(x, y)$.

Proof: Suppose that $B$ is a positive region consistent set of $S$ and $x, y \in U$ satisfy $\omega(x, y)$. Then $d(x) \neq d(y)$ and $x \in \operatorname{Pos}_{A}(d)$. By $x \in \operatorname{Pos}_{A}(d)=\operatorname{Pos}_{B}(d)$, we have $R_{B}(x) \subseteq[x]_{d}$. Because of $[x]_{d} \cap[y]_{d}=\varnothing$, it follows that $R_{B}(x) \cap[y]_{d}=\varnothing$, and consequently $y \notin R_{B}(x)$. Thus there exists $a \in B$ such that $(x, y) \notin R_{a}$, namely $a \in \alpha_{A}(x, y)$, and thus $B \cap \alpha_{A}(x, y) \neq \varnothing$.

Conversely, we suppose that $B \cap \alpha_{A}(x, y) \neq \varnothing$ for $x, y \in U$ satisfy $\omega(x, y)$. It only need to prove $\operatorname{Pos}_{A}(d) \subseteq \operatorname{Pos}_{B}(d)$. For any $x \in U$, if $x \notin \operatorname{Pos}_{B}(d)$, then $R_{B}(x) \not \subset[x]_{d}$. Thus there exists $y \in R_{B}(x)$ such that $y \notin[x]_{d}$. By $B \cap \alpha_{A}(x, y)=\varnothing$, we know that $x, y$ do not satisfy $\omega(x, y)$. It follows that $x \notin \operatorname{Pos}_{A}(d)$ by $d(x) \neq d(y)$. Thus $\operatorname{Pos}_{A}(d) \subseteq \operatorname{Pos}_{B}(d)$ as required.

This theorem shows that, with respect to positive region reduction, $x$ and $y$ need to be discerned if $x, y$ satisfy $\omega(x, y)$. In this case, we let $\vee \alpha_{A}(x, y)=\underset{a \in \alpha_{A}(x, y)}{\vee} a$ denote the disjunction of all attributes in $\alpha_{A}(x, y)$, where each attribute is looked upon as a Boolean variable. In what follows, $\Delta^{*}=\underset{(x, y) \in D^{*}}{\wedge} \vee \alpha_{A}(x, y)$ is called the positive discernibility function of $S$, where $D^{*}=\{(x, y) \in U \times U ; \omega(x, y)\}$. It is noted that $R_{A}$ is reflexive, therefore, $D^{*}$ need not to be symmetry in general.

Theorem 3.4 Let $S=(U, A, d)$ be a generalized decision system and $\Delta^{*}$ be the positive discernibility function of $S$. If

$$
\Delta^{* \prime}=\left(a_{11} \wedge \cdots \wedge a_{1 m_{1}}\right) \vee \cdots \vee\left(a_{k 1} \wedge \cdots \wedge a_{k m_{k}}\right)
$$

is the reduced disjunctive form of $\Delta^{*}$, then $\operatorname{Re} d=\left\{T_{1}, \cdots, T_{k}\right\}$ is the set of all positive region reductions of $S$, where $T_{i}=\left\{a_{i 1}, \cdots, a_{i m_{i}}\right\}$ for each $i \leq k$. 
Proof: (1) For any $i \leq k, T_{i}$ is a positive region reduction of $S$. In fact, if there exist $x, y \in U$ such that $x, y$ satisfy $\omega(x, y)$ and $T_{i} \cap \alpha_{A}(x, y)=\varnothing$, then we let all Boolean variable in $T_{i}$ be assigned 1 and the other Boolean variables be assigned 0 . It follows that $\Delta^{*}=0$ because $\vee \alpha_{A}(x, y)=0$ and $\Delta^{* \prime}=1$ because $a_{i 1} \wedge \cdots \wedge a_{i m_{i}}=1$. This contradicts the fact that $\Delta^{* \prime}$ is the disjunctive form of $\Delta^{*}$. Thus $T_{i}$ is a positive region consistent set.

We suppose that there exists a proper subset $T \subset T_{i}$ such that $T \cap \alpha_{A}(x, y) \neq \varnothing$ for any $(x, y) \in D^{*}$. By the property of Boolean function, there exist $j \leq k$ such that $T_{j} \subseteq T$. It follows that $T_{j} \subset T_{i}$. This contradicts the fact that $\Delta^{* \prime}$ is the reduced disjunctive form of $\Delta^{*}$. Thus $T_{i}$ is a positive region reduction of $S$.

(2) We suppose that $B$ is a reduction of $S$. It follows that $B \cap \alpha_{A}(x, y) \neq \varnothing$ for $(x, y) \in D^{*}$. It follows that there exist $i \leq k$ such that $T_{i} \subseteq B$. Because $T_{i}$ is a positive region consistent set, we have $T_{i} \subseteq B$. Thus, $\left\{T_{1}, \cdots, T_{k}\right\}$ is just the set of all positive region reductions of $S$.

If $R_{A}$ is reflexive and symmetric, then $\alpha_{A}(x, y)=\alpha_{A}(y, x)$ for any $x, y \in U$. Hence we have the following corollary.

Corollary 3.1 Let $S=(U, A, d)$ be a generalized decision system and $U=\left\{x_{1}, x_{2}, \cdots, x_{n}\right\}$. If $R_{a}$ is reflexive and symmetric for any $a \in A$, then the positive discernibility function of $S$ is

$$
\Delta^{*}=\underset{(x, y) \in D_{1}^{*}}{\wedge} \vee \alpha_{A}(x, y)
$$

where $D_{1}^{*}=\left\{\left(x_{i}, x_{j}\right) ; 1 \leq j<i \leq n, \omega_{1}\left(x_{i}, x_{j}\right)\right\}, \omega_{1}(x, y)$ represents the condition: $\left(x \in \operatorname{Pos}_{A}(d) \vee y \in \operatorname{Pos}_{A}(d)\right) \wedge d(x) \neq d(y)$.

Theorem 3.5 Let $S=(U, A, d)$ be a generalized decision system and $R_{a}$ an equivalence relation for any $a \in A$. If $x \in \operatorname{Pos}_{A}(d), y \notin \operatorname{Pos}_{A}(d)$ and $d(x)=d(y)$, then there exists $z \notin \operatorname{Pos}_{A}(d)$ such that $d(x) \neq d(z)$ and $\alpha_{A}(x, y)=\alpha_{A}(x, z)$.

Proof: It is trivial that $R_{A}$ is an equivalence relation on $U$. We use $[y]_{A}$ to denote $R_{A}(y)$. By $x \in \operatorname{Pos}_{A}(d), y \notin \operatorname{Pos}_{A}(d)$ we have $[x]_{A} \subseteq[x]_{d},[y]_{A} \not \subset[y]_{d}$. It follows that there exists $z \in[y]_{A}$ such that $z \notin[y]_{d}$. Thus $d(y) \neq d(z)$, and hence $d(x) \neq d(z)$. By $z \in[y]_{A}$, we have $(y, z) \in R_{a}$ for any $a \in A$. In consequence,

$$
\alpha_{A}(x, y)=\{a \in A ; a(x) \neq a(y)\}=\{a \in A ; a(x) \neq a(z)\}=\alpha_{A}(x, z) .
$$

Furthermore, by $z \in[y]_{A}$, it follows that $[z]_{A}=[y]_{A}$. Thus we have $z \notin \operatorname{Pos}_{A}(d)$ by $y \notin \operatorname{Pos}_{A}(d)$.

Remark: Let $S=(U, A, d)$ be a decision system and $R_{a}$ an equivalence relation for any $a \in A$.
Skowron[11] proposed the discernibility conditions for object pairs that need to discern with respect to positive region reduction. The discernibility conditions are

$$
\begin{aligned}
\omega_{S}(x, y): x \in \operatorname{Pos}_{A}(d) \wedge y \notin \operatorname{Pos}_{A}(d) ; \\
\quad \text { or } x \notin \operatorname{Pos}_{A}(d) \wedge y \in \operatorname{Pos}_{A}(d) ; \\
\text { or } x \in \operatorname{Pos}_{A}(d) \wedge y \in \operatorname{Pos}_{A}(d) \wedge d(x) \neq d(y) .
\end{aligned}
$$

According to above theorem, the object pair $(x, y)$ that satisfies $d(x)=d(y)$ do not need to discern in the criterion of positive region reduction. To be specific, Skowrons' discernibility conditions can be simplified as following:

$$
\omega_{1}(x, y):\left(x \in \operatorname{Pos}_{A}(d) \vee y \in \operatorname{Pos}_{A}(d)\right) \wedge d(x) \neq d(y) .
$$

In essence, based on Corollary 3.1, the discernibility condition is $\omega_{1}(x, y)$ when the indiscernibility relation satisfies reflexivity and symmetry.

Theorem 3.4 presents an approach to calculate the positive region reductions by discernibility function. Similarly as pointed out in [11], the approach is NP hard. In the following of this section, we present a heuristic algorithm based on discernibility matrix to calculate positive region reduction.

Let $S=(U, A, d)$ be a generalized decision system, $B \subseteq A$. By Theorem 3.3, $B$ is a positive region consistent set of $S$ if and only if $B \cap \alpha_{A}(x, y) \neq \varnothing$ for $x, y \in U$ satisfy $\omega(x, y)$. It follows that $D^{*}$ is the set of element pairs that needs to be discerned with respect to positive region reduction. For an attribute $a \in A,\left\{(x, y) \in D^{*} ; a \in \alpha_{A}(x, y)\right\}$ is the set of object pairs that $a$ can discern. Thus, the bigger the set $\left\{(x, y) \in D^{*} ; a \in \alpha_{A}(x, y)\right\}$, the more possible that $a$ is an element of a reduction. Based on this observation, we propose the notion of discernible degree.

Definition 3.3 Let $S=(U, A, d)$ be a generalized decision system, $E=\underset{(x, y) \in D^{*}}{\cup} \alpha_{A}(x, y)$. For any $a \in E$, the positive region discernible degree $\lambda(a)$ of $a$ is defined as

$$
\lambda(a)=\frac{\left|\left\{(x, y) \in D^{*} ; a \in \alpha_{A}(x, y)\right\}\right|}{\left|D^{*}\right|},
$$

where $\left|\left\{(x, y) \in D^{*} ; a \in \alpha_{A}(x, y)\right\}\right|$ and $\left|D^{*}\right|$ are cardinalities of $\left\{(x, y) \in D^{*} ; a \in \alpha_{A}(x, y)\right\}$ and $D^{*}$ respectively.

Intuitively speaking, the bigger the $\lambda(a)$, the more important the attribute $a$. We propose the following algorithm.

Algorithm 1

1) Input the generalized decision system $S=(U, A, d)$.

2) Compute the positive region $P_{A}(d)$ of $d$ and $\alpha_{A}(x, y)$ for every $(x, y) \in D^{*}$.

3) Place $\alpha_{A}(x, \dot{y})$ in discernibility matrix $D M_{1}$.

4) Compute the positive region discernible degree $\lambda(a)$ for each $a \in \underset{\alpha_{A}(x, y) \in D M_{1}}{\cup} \alpha_{A}(x, y)$, where 


$$
\lambda(a)=\frac{\left|\left\{\alpha_{A}(x, y) \in D M_{1} ; a \in \alpha_{A}(x, y)\right\}\right|}{\left|D M_{1}\right|} .
$$

5) Choose $a_{1}$ such that $\lambda\left(a_{1}\right)=\max _{b \in E} \lambda(b)$ (If there are more than one attributes with this property, then any one of the attribute may be chosen), delete $\alpha_{A}(x, y)$ which contain a from discernibility matrix $D M_{1}$ to obtain $D M_{2}$.

6) Go back to step 3 till $D M_{i+1}=\varnothing$. Then $T=\left\{a_{1}, \cdots, a_{i}\right\}$ is a positive region reduction.

Example 3.1 We consider the generalized decision system $S=(U, A, d)$, where $U=\left\{x_{1}, x_{2}, x_{3}, x_{4}\right\}, A=\{a, b, c\}$, the neighborhoods are given by:

$$
\begin{aligned}
& R_{a}\left(x_{1}\right)=\left\{x_{1}, x_{2}\right\}, R_{a}\left(x_{2}\right)=\left\{x_{2}, x_{3}, x_{4}\right\}, R_{a}\left(x_{3}\right)=\left\{x_{2}, x_{3}\right\}, \\
& R_{a}\left(x_{4}\right)=\left\{x_{3}, x_{4}\right\}, R_{b}\left(x_{1}\right)=\left\{x_{1}, x_{2}, x_{4}\right\}, R_{b}\left(x_{2}\right)=\left\{x_{2}, x_{3}\right\}, \\
& R_{b}\left(x_{3}\right)=\left\{x_{1}, x_{3}, x_{4}\right\}, R_{b}\left(x_{4}\right)=\left\{x_{4}\right\}, R_{c}\left(x_{1}\right)=\left\{x_{1}, x_{2}\right\}, \\
& R_{c}\left(x_{2}\right)=\left\{x_{2}, x_{3}, x_{4}\right\}, R_{c}\left(x_{3}\right)=\left\{x_{2}, x_{3}, x_{4}\right\}, R_{c}\left(x_{4}\right)=\left\{x_{4}\right\} .
\end{aligned}
$$

Furthermore, $U / d=\left\{D_{1}, D_{2}\right\}, D_{1}=\left\{x_{1}, x_{2}\right\}, D_{2}=\left\{x_{3}, x_{4}\right\}$. It follows that $R_{A}\left(x_{1}\right)=\left\{x_{1}, x_{2}\right\}, R_{A}\left(x_{2}\right)=\left\{x_{2}, x_{3}\right\}, R_{A}\left(x_{3}\right)=\left\{x_{3}\right\}$, $R_{A}\left(x_{4}\right)=\left\{x_{4}\right\}$. We note that $R_{A}$ is reflexive, but not symmetric and transitive. By routine computation, $\operatorname{Pos}_{A}(d)=\left\{x_{1}, x_{3}, x_{4}\right\}$,

$$
D M_{1}=\left(\begin{array}{ccccc} 
& x_{1} & x_{2} & x_{3} & x_{4} \\
x_{1} & & & \{a, b, c\} & \{a, c\} \\
x_{2} & & & & \\
x_{3} & \{a, c\} & \{b\} & & \\
x_{4} & \{a, b, c\} & \{a, b, c\} & &
\end{array}\right) .
$$

Thus $\lambda(a)=\frac{5}{6}, \lambda(b)=\frac{4}{6}, \lambda(c)=\frac{5}{6}$. Choose $a$, then

$$
D M_{2}=\left(\begin{array}{ccccc} 
& x_{1} & x_{2} & x_{3} & x_{4} \\
x_{1} & & & & \\
x_{2} & & & & \\
x_{3} & & \{b\} & & \\
x_{4} & & &
\end{array}\right),
$$

and choose $b$, then $D M_{3}=\varnothing$. Thus $T=\{a, b\}$ is a positive region reduction.

Note: If we firstly choose $c$, then we obtain another positive region reduction $T=\{b, c\}$.

\section{Distribution REDUCTIONS FOR GENERALIZED DECISION SYSTEMS}

Kryszkiewicz[3] proposed an rough set approach to incomplete information systems where the indiscernibility relation is a tolerance relation (reflexive and symmetric relation). In this section, we generalized the approach to generalized decision systems.

Let $S=(U, A, d)$ be a generalized decision system, $B \subseteq A$. We define $d_{B}: U \rightarrow P\left(V_{d}\right)$ as

$$
d_{B}(x)=d\left(R_{B}(x)\right)=\left\{d(y) ; y \in R_{B}(x)\right\} .
$$

Namely, $d_{B}(x)$ is the set of $d$ attribute values of objects in $R_{B}(x)$. The mapping $d_{B}$ is called decision function determined by $B$.

Definition 4.1 Let $S=(U, A, d)$ be a generalized decision system, $B \subseteq A$. If $d_{B}=d_{A}$, then $B$ is called a distribution consistent set of $S$, and the minimal distribution consistent set of $S$ (with respect to set inclusion relation) is called a distribution reduction of $S$.

Theorem 4.1[11] Let $S=(U, A, d)$ be a generalized decision system, $B \subseteq A, U / d=\left\{D_{1}, \cdots, D_{r}\right\}$. Then $B$ is a distribution consistent set if and only if $\overline{R_{B}}\left(D_{i}\right)=\overline{R_{A}}\left(D_{i}\right)$ for each $i \leq r$.

Theorem 4.2 Let $S=(U, A, d)$ be a generalized decision system, $B \subseteq A$. Then $B$ is a distribution consistent set if and only if $B \cap \alpha_{A}(x, y) \neq \varnothing$ for any $(x, y) \in D_{2}^{*}$, where $D_{2}^{*}=\left\{(x, y) ; d(y) \notin d_{A}(x)\right\}$.

Proof: Necessity: Notice that when $(x, y) \in D_{2}^{*}$, we have $d(y) \notin d_{A}(x)$ and hence $y \notin R_{A}(x), \alpha_{A}(x, y) \neq \varnothing$. Let $d_{B}=d_{A}$ and $(x, y) \in D_{2}^{*}$. By $d(y) \notin d_{A}(x)$ it follows that $d(y) \notin d_{B}(x)$. Thus $R_{B}(x) \cap[y]_{d}=\varnothing$, and $y \notin R_{B}(x)$. It follows that there exists $b \in B$ such that $(x, y) \notin R_{b}$, namely, $B \cap \alpha_{A}(x, y) \neq \varnothing$.

Sufficiency: For any $x \in U$, we have $d_{A}(x) \subseteq d_{B}(x)$. Suppose that $u$ is a decision value of $d, u \notin d_{A}(x)$ and $d(y)=u$. For any $z \in[y]_{d}$, it follows that $d(z)=d(y) \notin d_{A}(x)$, and hence $(x, z) \in D_{2}^{*}$. Consequently, we have $B \cap \alpha_{A}(x, z) \neq \varnothing$, namely, there exists $b \in B$ such that $(x, z) \notin R_{b}$, and thus $z \notin R_{B}(x)$. It follows that $R_{B}(x) \cap[y]_{d}=\varnothing$, and in consequence $u=d(y) \notin d\left(R_{B}(x)\right)=d_{B}(x)$. Thus $d_{B}(x) \subseteq d_{A}(x)$. It follows that $d_{B}=d_{A}$ and $B$ is a distribution consistent set of $S$ as required.

Let $S=(U, A, d)$ be a generalized decision system. In what follows, $\Delta^{0}=\underset{(x, y) \in D_{2}^{*}}{\wedge} \vee \alpha_{A}(x, y)$ is called the distribution discernibility function of $S$.

Corollary 4.1 Let $S=(U, A, d)$ be a generalized decision system. If $\Delta^{0 *}=\left(a_{11} \wedge \cdots \wedge a_{1 m_{1}}\right) \vee \cdots \vee\left(a_{k 1} \wedge \cdots \wedge a_{k m_{k}}\right)$ is the reduced disjunctive form of $\Delta^{0}$, then $\operatorname{Re} d=\left\{T_{1}, \cdots, T_{k}\right\}$ is the set of all distribution reductions of $S$, where $T_{i}=\left\{a_{i 1}, \cdots, a_{i m_{i}}\right\}$ for each $i \leq k$.

Theorem 4.2 and Corollary 4.1 show the method of distribution reduction based on generalized indiscernibility relation, which only satisfies reflexivity. Obviously, the methods improve the conclusion of literature. Similarly, We propose the following algorithm to compute distribution reduction.

Algorithm 2

1) Input the generalized decision system $S=(U, A, d)$.

2) Compute ${ }^{*}$ and $\alpha_{A}(x, y)$ for every $(x, y) \in D_{2}^{*}$.

3) Place $\alpha_{A}(x, y)$ in discernibility matrix $D M_{1}^{*}$. 
4) Compute the distribution discernible degree $\lambda(a)$ for each $a \in \underset{\alpha_{A}(x, y) \in D M_{1}^{*}}{\cup} \alpha_{A}(x, y)$, where

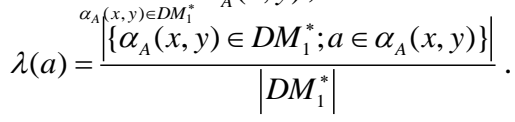

5) Choose $a_{1}$ such that $\lambda\left(a_{1}\right)=\max \lambda(b)$ (If there are more than one attributes with this property, then any one of the attribute may be chosen), delete $\alpha_{A}(x, y)$ which contain a from discernibility matrix $D M_{1}^{*}$ to obtain $D M_{2}^{*}$.

6) Go back to step 3 till $D M_{i+1}^{*}=\varnothing$. Then $T=\left\{a_{1}, \cdots, a_{i}\right\}$ is a distribution reduction.

The following theorem shows the connection between the concepts of distribution reduction and positive region reduction.

Theorem 4.3 Let $S=(U, A, d)$ be a generalized decision system, $B \subseteq A, U / d=\left\{D_{1}, \cdots, D_{r}\right\}$. If $B$ is a distribution consistent set, then $B$ is a positive region consistent set.

Proof: We suppose that $B$ is a distribution consistent set. It follows that $\overline{R_{B}}\left(D_{i}\right)=\overline{R_{A}}\left(D_{i}\right)$ for each $i \leq r$. Thus

$$
\begin{aligned}
& \underline{R_{B}}\left(D_{i}\right)=\sim \overline{R_{B}}\left(\sim D_{i}\right)=\sim \overline{R_{B}}\left(\cup_{j \neq i} D_{j}\right)=\sim \bigcup_{j \neq i}^{\cup \overline{R_{B}}}\left(D_{j}\right) \\
= & \sim \underset{j \neq i}{\cup} \overline{R_{A}}\left(D_{j}\right)=\sim \overline{R_{A}}\left(\cup_{j \neq i} D_{j}\right)=\sim \overline{R_{A}}\left(\sim D_{i}\right)=\underline{R_{A}}\left(D_{i}\right) .
\end{aligned}
$$

Consequently, $B$ is a positive region consistent set.

\section{CONCLUSIONS}

Rough set under incomplete information has been extensively studied. Researchers have put forward several similarity relations on objects and some attribute reduction approaches for incomplete information systems. This paper is devoted to the study of positive region reduction and distribution reduction based on generalized indiscernibility relation.

The judgment theorems for positive region reduction and distribution reduction of generalized decision systems and attribute reduction approaches are presented. Furthermore, the reduction algorithms based on discernible degree are proposed. Based on this work, we can further probe the rough set model under incomplete information and its application in knowledge discovery.

\section{REFERENCES}

[1] Z.Bonikowski, E.Bryniarski, U.Wybraniec, "Extensions and intentions in the rough set theory," Information Sciences, vol. 107, pp. 149-167, 1998.

[2] L.H.Guan, G.Y.Wang, Generalized approximations defined by nonequivalence relations, Information Sciences, vol. 193, pp. 163-179, 2012.

[3] M.Kryszkiewicz, Rough set approach to incomplete information system, Information Sciences, vol. 112, pp. 39-49, 1998.

[4] M.Kryszkiewicz, Properties of incomplete information systems in the framework of rough sets, Rough Sets in Data Mining and Knowledge Discovery, Physica-Verlag, 1998, pp. 422-450.

[5] K.Marzena K, Comparative study of alternative types of knowledge reduction in inconsistent systems, International Journal of Intelligent Systems, vol. 16, pp. 105-120, 2001.

[6] Z.Pawlak, Rough sets, Int. J. Computer and Information Sci., vol. 11, pp. 341-356, 1982.

[7] Z.Pawlak, A.Skowron, Rough sets: Some extensions, Information Sciences, vol. 177, pp. 28-40, 2007.

[8] K.Qin, Z.Pei, J.Yang, Y.Xu, Approximation operators on complete completely distributive lattices, Information Sciences, vol. 247, pp. 123130,2013

[9] K.Qin, H.Zhao, Z.Pei, The reduction of decision table based on generalized indiscernibility relation, Journal of Xihua University, vol.32(4), pp.1-4, 2013

[10] A.M.Radzikowska, E.E.Kerre, A comparative study of fuzzy rough sets, Fuzzy Sets and Systems, vol. 126, pp.137-155, 2002

[11] A.Skowron, C.Rauszer, The discernibility matrices and functions in information systems, In: R. Slowinski (Ed.), Intelligent Decision Support-Handbook of Applications and Advances of the Rough Sets Theory, Kluwer Academic Publishers, London, pp.331-362, 1992.

[12] J.Stefanowski, A.Tsoukias, Incomplete information tables and rough classification, Computational Intelligence, 17(3)2001, pp. 545-566

[13] G.Y.Wang, Extension of rough set under incomplete information systems, Journal of Computer Research and Development (in Chinese), vol. 39, pp. 1238-1243, 2002.

[14] G.Y.Wang, H.Yu, D.Yang, Decision table reduction based on conditional information entropy, Chinese Journal of Computers(in Chinese), vol. 25, pp. 759-766, 2002.

[15] Y.Y.Yao, Relational interpretation of neighborhood operators and rough set approximation operator, Information Sciences, 111, pp.239-259, 1998.

[16] Y.Y.Yao, Y.Zhao, Discernibility matrix simplification for constructing attribute reducts, Information Sciences, 179, pp.867-882, 2009.

[17] X.Yin, X.Jia, L.Shang, A new extension model of rough sets under incomplete information, Lecture Notes in Artificial Intelligence, 4062, pp. 141-146, 2006.

[18] X.H.Zhang, B.Zhou, P.Li, A general frame for intuitionistic fuzzy rough sets, Information Sciences, 216, pp.34-49, 2012.

[19] X.H.Zhang, J.H.Dai, Y.C.Yu, On the union and intersection operations of rough sets based on various approximation spaces, Information Sciences, 292, pp.214-229, 2015. 